\title{
INTERACTION BETWEEN THE FORCED AND PARAMETRIC EXCITATIONS WITH DIFFERENT DEGREES OF SMALLNESS
}

\author{
NGUYEN VAN DAO \\ Vietnam National University, Hanoi
}

\begin{abstract}
The nonlinear system under consideration in this paper has a specification which can be stated as an interaction between the first order of smallness nonresonance parametric excitation and the second order of smallness resonance forced excitation. In the first approximation these excitations have no effect. However, they do interact one with another in the second approximation.

The equations for the amplitude and phase of oscillation are found by means of the asymptotic method. The stationary oscillations and their stability are of special interest.
\end{abstract}

\section{The equation of motion and asymptotic solutions}

Let us consider a nonlinear system governed by the differential equation

$$
\ddot{x}+\omega^{2} x=\varepsilon p x \cos \omega t+\varepsilon^{2}\left[\Delta x-2 h \dot{x}-\beta x^{3}+r \cos (\omega t-\eta)\right],
$$

where

$$
\omega^{2}=1+\varepsilon^{2} \Delta,
$$

$\varepsilon$ is a small dimensionless parameter, 1 is natural frequency, $\Delta$ is detuning parameter, $p, h, \beta, r, \eta, \omega$ are constants and overdots denote differentiation with respect to time $t$.

We look for the solution of the equation (1.1) in the form:

$$
x=a \cos \theta+\varepsilon u_{1}(a, \psi, \theta)+\varepsilon^{2} u_{2}(a, \psi, \theta)+\ldots,
$$

where $\theta=\omega t+\psi, u_{i}(a, \psi, \theta)$ are periodic functions with period $2 \pi$ with respect to both angular variables $\psi$ and $\theta$, and $a$ and $\psi$ are functions of time which will be determined from the equations:

$$
\begin{aligned}
& \frac{d a}{d t}=\varepsilon A_{1}(a, \psi)+\varepsilon^{2} A_{2}(a, \psi)+\ldots \\
& \frac{d \psi}{d t}=\varepsilon B_{1}(a, \psi)+\varepsilon^{2} B_{2}(a, \psi)+\ldots
\end{aligned}
$$


these equations $A_{i}(a, \psi), B_{i}(a, \psi)$ are periodic functions of the angular variable with period $2 \pi$.

Substituting the expressions (1.3) and (1.4) into the equation (1.1) and comring the coefficient of $\varepsilon^{1}$ we obtain

$$
-2 \omega A_{1} \sin \theta-2 \omega a B_{1} \cos \theta+\omega^{2}\left(\frac{\partial^{2} u_{1}}{\partial \theta^{2}}+u_{1}\right)=a p \cos (\theta-\psi) \cos \theta
$$

Imparing the harmonics in (1.5) gives:

$$
\begin{aligned}
& A_{1}=B_{1}=0 \\
& u_{1}=\frac{p a}{2 \omega^{2}}\left[\cos \psi-\frac{1}{3} \cos (2 \theta-\psi)\right] .
\end{aligned}
$$

Comparing the coefficients of $\varepsilon^{2}$ in (1.1) we have

$$
\begin{aligned}
& -2 \omega A_{2} \sin \theta-2 \omega a B_{2} \cos \theta+\omega^{2}\left(\frac{\partial^{2} u_{2}}{\partial \theta^{2}}+u_{2}\right)=p u_{1} \cos \omega t+\Delta a \cos \theta \\
& +2 h \omega a \sin \theta-\beta a^{3} \cos ^{3} \theta+r \cos [\theta-(\psi+\eta)] .
\end{aligned}
$$

quating the coefficients of the first harmonics $\sin \theta$ and $\cos \theta$ in (1.7) we obtain

$$
\begin{aligned}
& A_{2}(a, \psi)=-h a-\frac{p^{2} a}{8 \omega^{3}} \sin 2 \psi-\frac{r}{2 \omega} \sin (\psi+\eta) \\
& B_{2}(a, \psi)=-\frac{\Delta}{2 \omega}-\frac{p^{2}}{12 \omega^{3}}+\frac{3 \beta}{8 \omega} a^{2}-\frac{p}{8 \omega^{3}} \cos 2 \psi-\frac{r}{2 \omega a} \cos (\psi+\eta),
\end{aligned}
$$

$2 \approx 1$

Thus, in the second approximation one has:

$$
x=a \cos \theta+\frac{\varepsilon p}{2 \omega^{2}}\left[\cos \psi-\frac{1}{3} \cos (2 \theta-\psi)\right], \quad \theta=\omega t+\psi
$$

here $a$ and $\psi$ satisfy the following differential equations:

$$
\begin{aligned}
& \frac{d a}{d t}=-\frac{\varepsilon^{2}}{2 \omega}\left[2 h \omega a+\frac{p^{2} a}{4} \sin 2 \psi+r \sin (\psi+\eta)\right] \\
& \frac{d \psi}{d t}=-\frac{\varepsilon^{2}}{2 \omega}\left[\Delta+\frac{p^{2}}{6}-\frac{3 \beta}{4} a^{2}+\frac{p^{2}}{4} \cos 2 \psi+\frac{r}{a} \cos (\psi+\eta)\right],
\end{aligned}
$$

$\neq 0$ 


\section{Stationary solutions}

Denoting

$$
C=\Delta+\frac{p^{2}}{6}-\frac{3}{4} \beta a_{0}^{2}, \quad D=\frac{p^{2}}{4}, \cdots=2 h \omega
$$

we have the following equations for stationary values $a_{0}, \psi_{0}$ satisfying the relations:

$$
\frac{d a}{d t}=\frac{d \psi}{d t}=0 ; \cdot f=g=0,
$$

where

$$
\begin{aligned}
& f=H a_{0}+D a_{0} \sin 2 \psi_{0}+r \sin \left(\psi_{0}+\eta\right), \\
& g=C a_{0}+D a_{0} \cos 2 \psi_{0}+r \cos \left(\psi_{0}+\eta\right)
\end{aligned}
$$

We transform equations (2.2) into two equivalent ones:

$$
\begin{aligned}
& f \cos \psi_{0}-g \sin \psi_{0}=(D-C) a_{0} \sin \psi_{0}+H a_{0} \cos \psi_{0}+r \sin \eta=0 \\
& f \sin \psi_{0}+g \cos \psi_{0}=H a_{0} \sin \psi_{0}+(D+C) a_{0} \cos \psi_{0}+r \cos \eta=0
\end{aligned}
$$

The condition for reality of $\sin \psi_{0}$ and $\cos \psi_{0}$ is $[2,3]$ :

$$
\begin{aligned}
& a_{0}^{2}\left[(D-C)^{2}+H^{2}\right] \geq r^{2} \sin ^{2} \eta, \\
& a_{0}^{2}\left[(D+C)^{2}+H^{2}\right] \geq r^{2} \cos ^{2} \eta .
\end{aligned}
$$

a) Supposing that

$$
M=D^{2}-\left(H^{2}+C^{2}\right) \neq 0,
$$

we have from equations (2.4):

$$
\begin{aligned}
\sin \psi_{0} & =\frac{r[H \cos \eta-(D+C) \sin \eta]}{\left[D^{2}-\left(H^{2}+C^{2}\right)\right] a_{0}} \\
\cos \psi_{0} & =\frac{r[H \sin \eta-(D-C) \cos \eta]}{\left[D^{2}-\left(H^{2}+C^{2}\right)\right] a_{0}}
\end{aligned}
$$

Eliminating $\psi_{0}$ we obtain:

$$
W\left(a_{0}, \omega\right)=0
$$

where

$$
W=a_{0}^{2}\left[D^{2}-\left(H^{2}+C^{2}\right)\right]^{2}-r^{2}\left[H^{2}+D^{2}+C^{2}-2 D C \cos 2 \eta-2 H D \sin 2 \eta\right] .
$$


b) If

$$
M=D^{2}-\left(H^{2}+C^{2}\right)=0,
$$

i.e. if the resonance curve takes the form

$$
C= \pm \sqrt{D^{2}-H^{2}}
$$

or

$$
\frac{3}{4} \beta a_{0}^{2}=\delta \pm \sqrt{\left(\frac{p^{2}}{4}\right)^{2}-4 h^{2} \omega^{2}}, \quad \delta=\Delta+\frac{p^{2}}{6},
$$

then by (2.8) one should have

$$
N_{1}=H \cos \eta-(D+C) \sin \eta=0, \quad N_{2}=H \sin \eta-(D-C) \cos \eta=0,
$$

or equivalently,

$$
N_{1} \cos \eta+N_{2} \sin \eta=0, \quad N_{1} \sin \eta-N_{2} \cos \eta=0 .
$$

These relations give:

$$
H=D \sin 2 \eta, \quad C=D \cos 2 \eta .
$$

Substituting these values into (2.5) and (2.6) we obtain the following restriction to the amplitude $a_{0}$ :

$$
a_{0}^{2} \geq \frac{r^{2}}{4 D^{2}} .
$$

Note. As it will be seen later, the curve (2.11) serves as the boundary of the stability zone.

\section{System without friction}

Now, let us consider a special case when $h=0$ and the equations (2.4) have the form:

$$
\begin{aligned}
& (D-C) a_{0} \sin \psi_{0}=-r \sin \eta, \\
& (D+C) a_{0} \cos \psi_{0}=-r \cos \eta
\end{aligned}
$$

a) If $D-C \neq 0$ and $D+C \neq 0$, then the resonance curve $C_{1}$ is determined by the equation of type (2.10) with $H=0$ :

$$
W\left(\omega^{2}, a_{0}^{2}\right)=0,
$$

where

$$
W\left(\omega^{2}, a_{0}^{2}\right)=a_{0}^{2}\left(D^{2}-C^{2}\right)^{2}-r^{2}\left(D^{2}+C^{2}-2 D C \cos 2 \eta\right) .
$$


In a particular case, when $\eta=0, \pi$ the resonance curve $C_{1}$ degenerates into

1) The curve $C_{1}^{1}: D=C$ (double)

2) The curve $C_{1}^{3}: a_{0}^{2}(D+C)^{2}-r^{2}=0$.

When $\eta=\frac{\pi}{2}, \frac{3 \pi}{2}$ the resonance curve $C_{1}$ degenerates into

3) The curve $C_{1}^{4}: \quad D=-C$ (double)

4) The curve $C_{1}^{6}: a_{0}^{2}(D-C)^{2}-r^{2}=0$.

b) If $D-C=0$ (the resonance curve $C_{2}$ ), then from (3.1) we have

$0 . a_{0} \sin \psi_{0}=-r \sin \eta \Rightarrow \sin \eta=0 \Rightarrow \eta=0, \pi$,

$2 D a_{0} \cos \psi_{0}=-r \cos \eta= \pm r \Rightarrow \psi_{0}=\arccos \left( \pm \frac{r}{2 D a_{0}}\right) \Rightarrow a_{0}^{2} \geq \frac{r^{2}}{4 D^{2}}$.

c) If $D+C=0$ (the resonance curve $C_{3}$ ), then from (3.1) we have

$$
\begin{aligned}
0 . a_{0} \cos \psi_{0} & =-r \cos \eta \Rightarrow \cos \eta=0 \Rightarrow \eta=\frac{\pi}{2}, \frac{3 \pi}{2} \\
2 D a_{0} \sin \psi_{0} & =-r \sin \eta= \pm r \Rightarrow \psi_{0}=\arcsin \left( \pm \frac{r}{2 D a_{0}}\right) \Rightarrow a_{0}^{2} \geq \frac{r^{2}}{4 D^{2}}
\end{aligned}
$$

\section{Stability of stationary oscillations}

With the notation (2.1) the equations (1.10) can be written in the form:

$$
\begin{aligned}
\frac{d a}{d t} & =-\frac{\varepsilon^{2}}{2 \omega}[H a+D a \sin 2 \psi+r \sin (\psi+\eta)], \\
a \frac{d \psi}{d t} & =-\frac{\varepsilon^{2}}{2 \omega}[C a+D a \cos 2 \psi+r \cos (\psi+\eta)] .
\end{aligned}
$$

To study the stability of stationary oscillations with amplitude $a_{0}$ and phase $\psi_{0}$ determined from equations $(2.2)$ or $(2.4)$ we introduce the variations:

$$
\tilde{a}=a-a_{0}, \quad \tilde{\psi}=\psi-\psi_{0}
$$

Substituting these values into $(4.1)$ we obtain

$$
\begin{aligned}
\frac{d \tilde{a}}{d t} & =-\frac{\varepsilon^{2}}{2 \omega}\left\{\left(H+D \sin 2 \psi_{0}\right) \widetilde{a}+\left[2 D a_{0} \cos 2 \psi_{0}+r \cos \left(\psi_{0}+\eta\right)\right] \widetilde{\psi}\right\} \\
a_{0} \frac{d \tilde{\psi}}{d t} & =-\frac{\varepsilon^{2}}{2 \omega}\left\{\left(C+C^{\prime} a_{0}+D \cos 2 \psi_{0}\right) \tilde{a}-\left[2 D a_{0} \sin 2 \psi_{0}+r \sin \left(\psi_{0}+\eta\right)\right] \tilde{\psi}\right\}
\end{aligned}
$$


where $C^{\prime}=-\frac{3}{2} \beta a_{0}$

The characteristic equation for last two equations is

$$
a_{0} \lambda^{2}+\frac{\varepsilon^{2}}{2 \omega} H_{*} \lambda-\frac{\varepsilon^{4}}{4 \omega^{2}} S=0
$$

where $\lambda$ is characteristic numbers,

$$
\begin{aligned}
H_{*}= & a_{0}\left[H-D \sin 2 \psi_{0}-r \sin \left(\psi_{0}+\eta\right)\right]=4 h \omega a_{0}>0, \\
S= & \left(H+D \sin 2 \psi_{0}\right)\left[2 D a_{0} \sin 2 \psi_{0}+r \sin \left(\psi_{0}+\eta\right)\right] \\
& +\left(C+C^{\prime} a_{0}+D \cos 2 \psi_{0}\right)\left[2 D a_{0} \cos 2 \psi_{0}+r \cos \left(\psi_{0}+\eta\right)\right] .
\end{aligned}
$$

The expression for $S$ can be written as

$$
S=a_{0}\left(D^{2}-H^{2}-C^{2}-a_{0} C C^{\prime}\right)+a_{0}^{2} C^{\prime} D \cos 2 \psi_{0}
$$

From (2.2) and (2.3) it follows:

$$
D a_{0} \cos 2 \psi_{0}=-C a_{0}-r\left(\cos \psi_{0} \cos \eta-\sin \psi_{0} \sin \eta\right)
$$

Substituting here the expressions $\cos \psi_{0}$ and $\sin \psi_{0}$ from (2.8) we obtain

$$
D a_{0} \cos 2 \psi_{0}=-C a_{0}-\frac{r^{2}}{a_{0}\left(D^{2}-H^{2}-C^{2}\right)}(C-D \cos 2 \eta) .
$$

Thus, we have

$$
\begin{gathered}
2\left(D^{2}-H^{2}-C^{2}\right) S=2 a_{0}\left(D^{2}-H^{2}-C^{2}\right)^{2}-4 a_{0}^{2} C C^{\prime}\left(D^{2}-H^{2}-C^{2}\right) \\
-2 r^{2} C C^{\prime}+2 r^{2} D C^{\prime} \cos 2 \eta=\frac{\partial W}{\partial a_{0}}, \\
S=\frac{1}{2\left(D^{2}-H^{2}-C^{2}\right)} \cdot \frac{\partial W}{\partial a_{0}}, \quad\left(D^{2}-H^{2}-C^{2}\right) \neq 0 .
\end{gathered}
$$

Thus, the stability condition of the stationary solutions $a_{0}$ and $\psi_{0}$ takes the form

$$
\begin{aligned}
& M \frac{\partial W}{\partial a_{0}}>0 \\
& M=H^{2}+C^{2}-D^{2}
\end{aligned}
$$

The resonance curve $(W=0)$ divides the plane $\left(a_{0}, \omega\right)$ into regions, in each of which the expression $W$ has a definite sign ( + or - ). If moving up along the 
straight line parallel to the axis $a_{0}$, we pass from a region $W<0$ to a region $W>0$, then at the point of intersection between the straight line and the resonance curve the derivative $\partial W / \partial a_{0}$ is positive. So, this point corresponds to a stable state of oscillation if $M>0$ and to an unstable one if $M<0$. On the contrary, if we pass from a region $W>0$ to a region $W<0$, then the point of intersection corresponding to a stable state of oscillation if $M<0$ and to an unstable one if $M>0$.

This work was financially supported by the Council for Natural Sciences of Vietnam.

\section{References}

1. Mitropolski Yu. A., Nguyen Van Dao. Applied asymptotic methods in nonlinear oscillations. Kluwer Academic Publishers, 1997.

2. Nguyen Van Dao. Interaction of the elements characterizing the quadratic nonlinearity and forced excitation with the other excitations. J. of Mechanics No 4, 1997.

3. Nguyen Van Dao. Interaction between the elements characterizing the forced and parametric excitations. Vietnam Journal of Mechanics, No 1, 1998.

Received November 15, 1997

\section{TƯONG TÁC GIŨ̃ CÁC KÍCH ĐộNG THÔNG SỐ VÀ CUÕ̃NG BỨC CÓ BẬC BÉ KHÁC NHAU}

Sự tương tác giữa kích động thông số không cộng hưởng có độ bé bậc một với kích động cưỡng bức cộng hường có độ bé bậc hai đã được khào sát. Ở xấp xỉ thứ nhất các kích động này không gây ra hiệu quá. Song chúng tương tác lẫn nhau trong xấp xî thứ hai. Các dao động dừng và sự ôn định của chúng đã được đặc biệt quan tâm nghiên cứu. 